\title{
DISCOVERY OF TWO NEW CLASS II METHANOL MASER TRANSITIONS IN G 345.01+1.79
}

\author{
S. P. Ellingsen ${ }^{1}$, A. M. Sobolev ${ }^{2}$, D. M. CragG $^{3}$, And P. D. Godfrey ${ }^{3}$ \\ ${ }^{1}$ School of Mathematics and Physics, University of Tasmania, Private Bag 37, Hobart, TAS 7001, Australia; Simon.Ellingsen@utas.edu.au \\ ${ }^{2}$ Ural Federal University, Lenin Avenue 51, 620000 Ekaterinburg, Russia \\ ${ }^{3}$ School of Chemistry, Building 23, Monash University, Victoria 3800, Australia \\ Received 2012 September 18; accepted 2012 September 24; published 2012 October 9
}

\begin{abstract}
We have used the Swedish ESO Submillimetre Telescope (SEST) to search for new class II methanol maser transitions toward the southern source G 345.01+1.79. Over a period of 5 days, we observed 11 known or predicted class II methanol maser transitions. Emission with the narrow line width and characteristic velocity of class II methanol masers (in this source) was detected in eight of these transitions, two of which have not previously been reported as masers. The new class II methanol maser transitions are the $13_{-3}-12_{-4} \mathrm{E}$ transition at $104.1 \mathrm{GHz}$ and the $5_{1}-4_{2} \mathrm{E}$ transition at $216.9 \mathrm{GHz}$. Both of these are from transition series for which there are no previous known class II methanol maser transitions. This takes the total number of known class II methanol maser series to 10, and the total number of transitions (or transition groups) to 18 . The observed $104.1 \mathrm{GHz}$ maser suggests the presence of two or more regions of masing gas with similar line of sight velocities, but quite different physical conditions. Although these newly discovered transitions are likely to be relatively rare, where they are observed, combined studies using the Australia Telescope Compact Array and the Atacama Large Millimeter Array offer the prospect to be able to undertake multi-transition methanol maser studies with unprecedented detail.
\end{abstract}

Key words: ISM: molecules - masers - radio lines: ISM - stars: formation

\section{INTRODUCTION}

Class II methanol masers are unique tracers of the early stages of high-mass star formation (see, e.g., Ellingsen 2006). The other maser transitions commonly observed in high-mass star formation regions (main-line $\mathrm{OH}$ and $22 \mathrm{GHz}$ water) are also observed in different environments, such as late-type stars and low-mass star formation regions. In contrast, despite a number of sensitive and extensive searches, no class II methanol masers have been found associated with anything other than high-mass star formation regions (Ellingsen et al. 1996; Minier et al. 2003; Xu et al. 2008). The majority of class II methanol maser sources are not associated with ultracompact H II (UCH II) regions (Phillips et al. 1998; Walsh et al. 1998). This indicates that either the masers are associated with objects that are not massive enough to form detectable $\mathrm{H}_{\text {II }}$ regions or that they are associated with high-mass stars prior to the formation of a UCH II region. Numerous observations at millimeter through mid-infrared wavelengths (e.g., Walsh et al. 2003; Ellingsen 2006; Cyganowski et al. 2009) suggest that many class II methanol maser sources are associated with a very early evolutionary phase in the high-mass star formation process.

Hindered rotation in the methanol molecule leads to a very large number of rotational and vibrational transitions in the millimeter and the submillimeter regime. A large number of these transitions have been observed in interstellar space and a select few show maser action. The transitions in which masers are observed have been empirically classified into two groups (Batrla et al. 1987; Menten 1991a). The class II methanol masers are closely associated with sites of high-mass star formation and related objects, including infrared sources, $\mathrm{H}$ II regions, and often $\mathrm{OH}$ or water masers. The $6.7 \mathrm{GHz} 5_{1}-6_{0} \mathrm{~A}^{+}$transition is the strongest and most common class II maser transition, having been observed toward more than 900 regions within our Galaxy (Caswell et al. 2010, 2011; Green et al. 2010). Class I methanol masers are found near high-mass star formation regions, but offset from the $\mathrm{H}$ II regions by a few tenths of a parsec (Kurtz et al. 2004). The $44.0 \mathrm{GHz} 7_{0}-6_{1} \mathrm{~A}^{+}$transition is the strongest and most common class I maser transition. This Letter focuses solely on class II methanol masers and hereafter it is implicit that all references to "methanol masers" refer to class II masers.

To date, 18 different transitions (or transition groups) associated with 8 different methanol transition series have been observed as class II methanol masers and their frequencies along with the discovery reference are listed in Table 1 . Counting the total number of known class II methanol maser transitions is somewhat subjective. The distinction we have made is that where there are two or more transitions at similar frequencies which are always (or nearly always) either present or absent together from sources and we have considered these to be a transition group. For example, all observations to date of the $38.3\left(6_{2}-5_{3} \mathrm{~A}^{-}\right)$and $38.5 \mathrm{GHz}\left(6_{2}-5_{3} \mathrm{~A}^{+}\right)$transitions show that where emission from one of these is detected, the other is also detected and with similar intensity (Haschick et al. 1989; Ellingsen et al. 2011). Hence, we consider the 38.3 and $38.5 \mathrm{GHz}$ masers to represent a single transition group. In contrast, the 6.7, 107.0, and $156.6 \mathrm{GHz}$ transitions are all members of the $J_{1}-(J+1)_{0} \mathrm{~A}^{+}$series; however, many sources that show emission in the $6.7 \mathrm{GHz}$ transition are not observed in either of the other two, so we count these as three separated maser transitions. The $J_{0}-J_{-1}$ E series at frequencies near $157 \mathrm{GHz}$ is another example of a single transition group (Slysh et al. 1995), although the $148.1 \mathrm{GHz} 15_{0}-15_{-1} \mathrm{E}$ is counted as a separate transition as it stands apart both in frequency and in the physical conditions it requires.

The two lowest frequency methanol maser transitions $\left(5_{1}-6_{0} \mathrm{~A}^{+}\right.$at $6.7 \mathrm{GHz}$ and $2_{0}-3_{-1} \mathrm{E}$ at $\left.12.2 \mathrm{GHz}\right)$ have been detected toward a large number of sources, but the majority of the other transitions are only observed toward a handful of sources (see Ellingsen et al. 2011 and references therein). Current models of class II methanol masers (Sobolev et al. 1997a; Cragg et al. 2002, 2005) find that the 6.7 and $12.2 \mathrm{GHz}$ transitions are strongly inverted over a wide range of physical 
Table 1

Class II Methanol Maser Transitions

\begin{tabular}{|c|c|c|c|c|}
\hline $\begin{array}{l}\text { Rest } \\
\text { Frequency }\end{array}$ & Transition & Series & $\begin{array}{l}\text { Discovery } \\
\text { Reference }\end{array}$ & $\begin{array}{c}\text { G } 345.01+1.79 \\
\text { Reference }\end{array}$ \\
\hline$\overline{6.7}$ & $5_{1}-6_{0} A^{+}$ & 1 & Menten (1991b) & Norris et al. (1993) \\
\hline 12.2 & $2_{0}-3_{-1} \mathrm{E}$ & 2 & Batrla et al. (1987) & Norris et al. (1988) \\
\hline 20.0 & $2_{1}-3_{0} \mathrm{E}$ & 3 & Wilson et al. (1985) & Ellingsen et al. (2004) \\
\hline 23.1 & $9_{2}-10_{1} \mathrm{~A}^{+}$ & 4 & Wilson et al. (1984) & Cragg et al. (2004) \\
\hline 29.0 & $8_{2}-9_{1} \mathrm{~A}^{-}$ & 4 & Wilson et al. (1993) & \\
\hline 37.7 & $7_{-2}-8_{-1} \mathrm{E}$ & 5 & Haschick et al. (1989) & Ellingsen et al. (2011) \\
\hline $38.3 / 38.5$ & $6{ }_{2}-5_{3} \mathrm{~A}^{-} / \mathrm{A}^{+}$ & 6 & Haschick et al. (1989) & Ellingsen et al. (2011) \\
\hline 85.6 & $6-2-7-1 \mathrm{E}$ & 5 & Cragg et al. (2001) & Cragg et al. (2001) \\
\hline $86.6 / 86.9$ & $7_{2}-6_{3} A^{-} / A^{+}$ & 6 & Cragg et al. (2001) & Cragg et al. (2001) \\
\hline 107.0 & $3_{1}-4_{0} \mathrm{~A}^{+}$ & 1 & Val'tts et al. (1995) & Caswell et al. (2000) \\
\hline 108.9 & $0_{0}-1_{-1} \mathrm{E}$ & 2 & Val'tts et al. (1999) & Val'tts et al. (1999) \\
\hline 148.1 & $15_{0}-15_{-1} \mathrm{E}$ & 7 & Salii \& Sobolev (2006) & Salii \& Sobolev (2006) \\
\hline 156.6 & $2{ }_{1}-3_{0} \mathrm{~A}^{+}$ & 1 & Slysh et al. (1995) & Caswell et al. (2000) \\
\hline 157.3 & $J_{0}-J_{-1}$ E group & 7 & Slysh et al. (1995) & Slysh et al. (1995) \\
\hline 165.0 & $J_{1}-J_{0} \mathrm{E}$ group & 8 & Salii et al. (2003) & Salii et al. (2003) \\
\hline 231.3 & $10_{2}-9 \mathrm{~A}^{-}$ & 6 & Salii \& Sobolev (2006) & Salii \& Sobolev (2006) \\
\hline
\end{tabular}

Notes. The rest frequencies have been rounded at the $100 \mathrm{kHz}$ significance level. The $\mathrm{G} 345.01+1.79$ reference is the most sensitive high-resolution observation available for the specific transition.

conditions, explaining both their high-brightness temperatures and common occurrence. The models successfully predict masing in all of the other class II transitions for which it has been observed, but generally the physical conditions implied are much more restricted (Sobolev et al. 1997b). So the presence of multiple class II maser transitions within one source can, through comparison with models, be used to constrain the physical conditions in the masing region (under the assumption that the various transitions all arise from the same location).

\section{OBSERVATIONS}

The observations were made using the Swedish ESO Submillimetre Telescope (SEST) between 2000 June 22 and 27. We observed a total of 13 different methanol transitions, 7 of which are known class II methanol maser transitions and the remaining 6 of which are predicted class II methanol maser transitions (Sobolev et al. 1997b). The frequencies of the observed transitions range from 84 to $265 \mathrm{GHz}$. These transitions fall into 11 different transition groups, as the $6{ }_{2}-5_{3} \mathrm{~A}^{-} / \mathrm{A}^{+}$and $10_{2}-9_{3} \mathrm{~A}^{-} / \mathrm{A}^{+}$represent a single transition group in each case. All of the observations were targeted toward the class II methanol maser source G 345.01+1.79 (more precisely G 345.010+1.792) at $\alpha_{\mathrm{J} 2000}=16^{\mathrm{h}} 56^{\mathrm{m}} 47^{\mathrm{s}} .58 ; \delta_{\mathrm{J} 2000}=$ $-40^{\circ} 14^{\prime} 25^{\prime \prime} .8$ (Caswell 2009). The on-source integration time for each of these transitions ranged from 30 to 60 minutes and the resulting rms per spectral channel ranged from 0.3 to $2.3 \mathrm{Jy}$. The observations were made by frequency switching with a throw of $6 \mathrm{MHz}$ and a switching time of 2 minutes. The spectra were obtained using the high-resolution acousto-optic spectrometer, which has a total bandwidth of $86 \mathrm{MHz}$ and a spectral resolution of $80 \mathrm{kHz}$, resulting in velocity resolutions of between 0.28 and $0.09 \mathrm{~km} \mathrm{~s}^{-1}$ (for the lowest and highest frequency transitions observed, respectively). The intensity scale was converted from Kelvin to Jy by assuming a sensitivity of $25 \mathrm{Jy} \mathrm{K}^{-1}$ for the transitions at frequencies less than $150 \mathrm{GHz}, 30 \mathrm{Jy} \mathrm{K}^{-1}$ for transitions at frequencies between 150 and $200 \mathrm{GHz}$, and $41 \mathrm{Jy} \mathrm{K}^{-1}$ for transitions at frequencies higher than $200 \mathrm{GHz}$.

The data were processed using Per Bergman's XS package, following the standard procedure for radio-millimeter single
Table 2

Summary of Lines Observed toward G 345.01+1.79

\begin{tabular}{lcccccc}
\hline \hline Transition & $\begin{array}{c}v \\
(\mathrm{MHz})\end{array}$ & $\begin{array}{c}\text { rms } \\
(\mathrm{Jy})\end{array}$ & $\begin{array}{c}T_{\text {sys }} \\
(\mathrm{K})\end{array}$ & $\begin{array}{c}\text { Integration } \\
(\text { minutes })\end{array}$ & $\begin{array}{c}\text { Peak Flux } \\
(\mathrm{Jy})\end{array}$ & $\begin{array}{c}\text { Velocity } \\
\left(\mathrm{km} \mathrm{s}^{-1}\right)\end{array}$ \\
\hline $13_{-3}-14_{-2} \mathrm{E}$ & 84423.769 & 0.4 & 143 & 50 & $<1.9$ & \\
$6_{-2}-7_{-1} \mathrm{E}$ & 85568.084 & 0.4 & 160 & 54 & $10.9(1.9)$ & -22.1 \\
$7_{2}-6_{3} \mathrm{~A}^{-}$ & 86615.578 & 0.3 & 149 & 42 & $7.2(0.6)$ & -22.3 \\
$7_{2}-6_{3} \mathrm{~A}^{+}$ & 86902.956 & 0.3 & 140 & 60 & $6.8(0.5)$ & -22.1 \\
$13_{-3}-12_{-4} \mathrm{E}$ & 104060.647 & 0.7 & 254 & 30 & $2.8(1.1)$ & -22.3 \\
$3_{1}-4_{0} \mathrm{~A}^{+}$ & 107013.812 & 0.7 & 265 & 56 & $66(8)$ & -21.9 \\
$0_{0}-1_{-1} \mathrm{E}$ & 108893.948 & 0.3 & 166 & 60 & $8.3(0.4)$ & -21.7 \\
$2_{1}-3_{0} \mathrm{~A}^{+}$ & 156602.413 & 1.3 & 600 & 56 & $27.6(1.2)$ & -21.8 \\
$7_{0}-7-1 \mathrm{E}$ & 156828.533 & 2.3 & 1015 & 54 & $45.0(1.8)$ & -21.7 \\
$5_{1}-4_{2} \mathrm{E}^{-}$ & 216945.600 & 0.8 & 203 & 60 & $4.2(0.8)$ & -22.5 \\
$10_{2}-9_{3} \mathrm{~A}^{-}$ & 231281.100 & 1.5 & 267 & 42 & $<7.5$ & \\
$10_{2}-9_{3} \mathrm{~A}^{+}$ & 232418.590 & 1.6 & 329 & 30 & $<7.8$ & \\
$4_{2}-5_{1} \mathrm{~A}^{-}$ & 234683.390 & 1.3 & 315 & 50 & $<6.4$ & \\
\hline
\end{tabular}

Note. The rest frequencies for transitions $<200 \mathrm{GHz}$ are from Tsunekawa et al. (1995) and the remainder are from Sastry et al. (1984).

dish spectral line observations. The details of the transitions observed and the achieved sensitivities are summarized in Table 2. Because we were searching for narrow maser lines, the spectra were not smoothed prior to measuring the rms.

\section{RESULTS}

Emission was detected at 9 of the 13 methanol transitions observed. These observations represent the first astronomical detection of maser emission from the $104.1 \mathrm{GHz}\left(13_{-3}-12_{-4} \mathrm{E}\right)$ transition and the $216.9 \mathrm{GHz}\left(5_{1}-4_{2} \mathrm{E}\right)$ transition. The spectra of all of the methanol maser transitions detected during the current SEST observations are shown in Figure 2. It should be noted that the class II methanol maser reported here is a different transition from the $104.3 \mathrm{GHz}\left(11_{-1}-10_{-2}\right.$ E) class I methanol maser previously discovered by Voronkov et al. (2005). Spectra of the emission in these two new class II methanol maser transitions are shown in Figure 1. In both cases the maser emission consists of a narrow (FWHM $<0.5 \mathrm{~km} \mathrm{~s}^{-1}$ ) peak at a velocity of around $-22 \mathrm{~km} \mathrm{~s}^{-1}$. This is the approximate velocity 

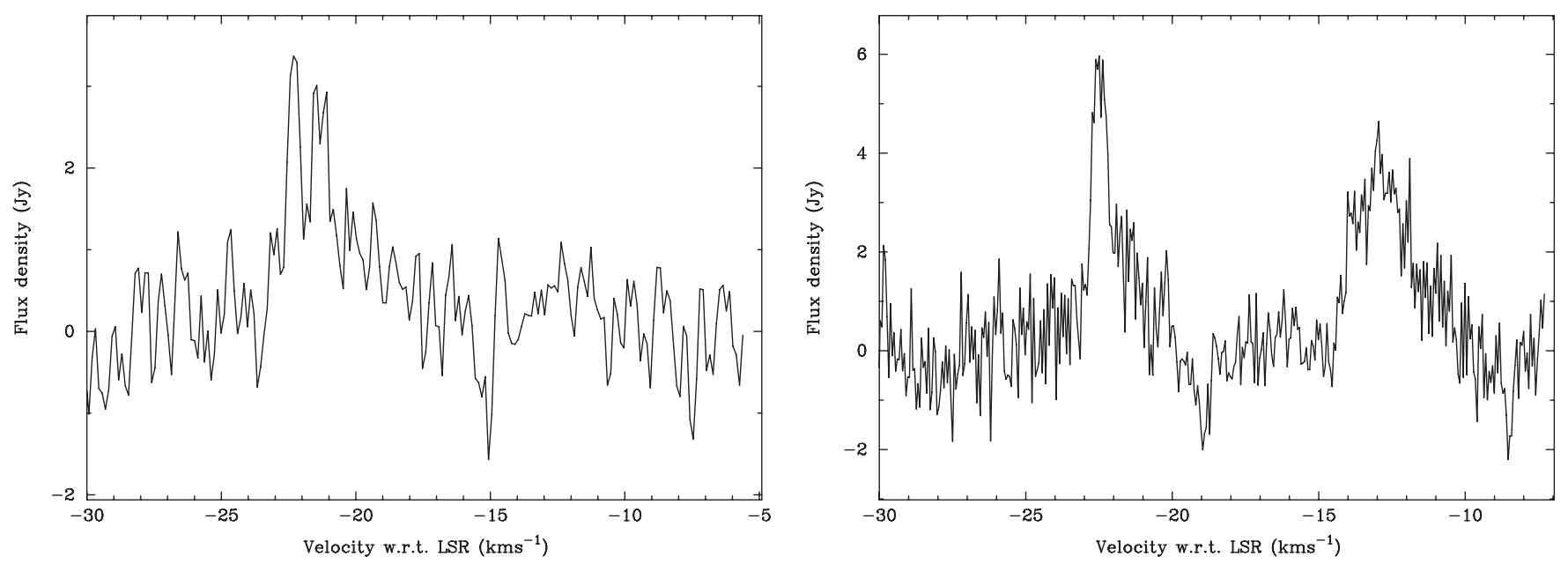

Figure 1. Spectra of the $104.1 \mathrm{GHz}\left(13_{-3}-12_{-4}\right.$ E) (left) and $216.9 \mathrm{GHz}\left(5_{1}-4_{2} \mathrm{E}\right)$ (right) transitions of methanol toward G $345.01+1.79$.

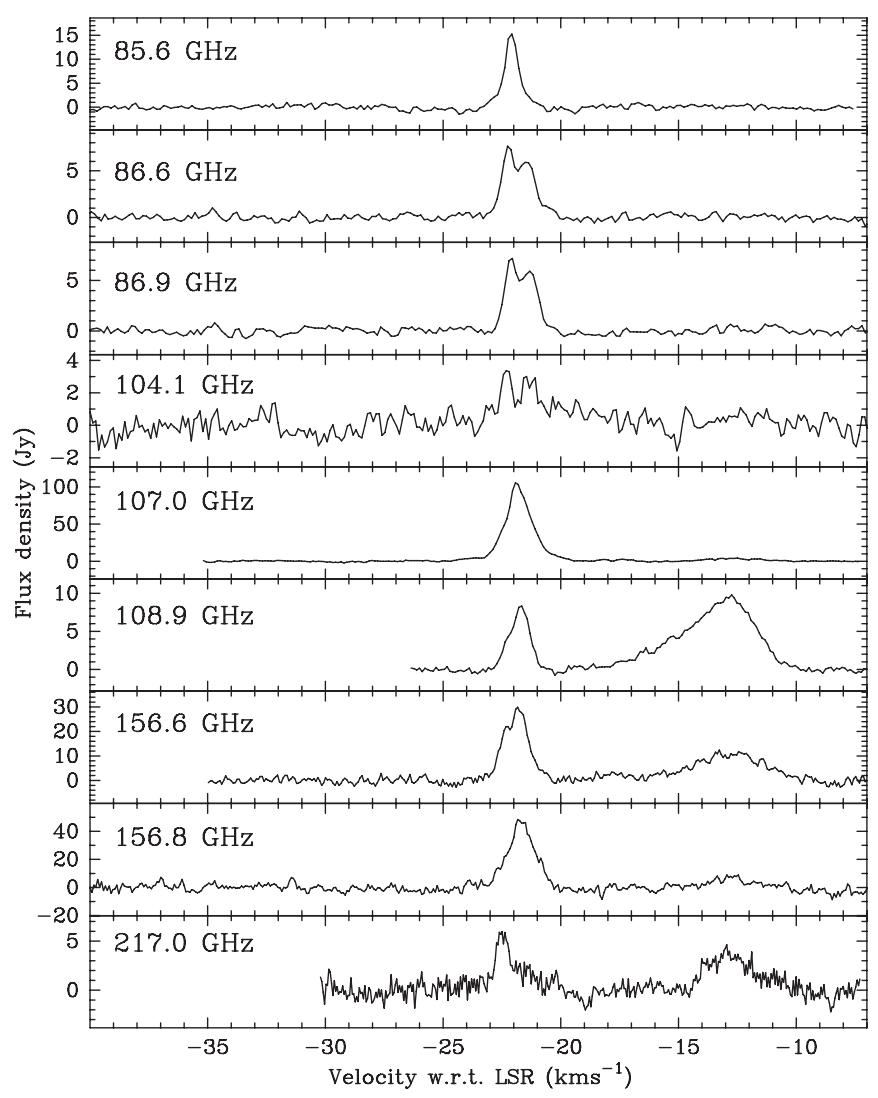

Figure 2. Spectra of the detected class II methanol maser transitions toward $\mathrm{G} 345.01+1.79$.

at which most of the rare class II methanol masers observed toward G 345.01+1.79 peak. Observations of the $104.1 \mathrm{GHz}$ transition of methanol have previously been made toward the archetypal class II methanol maser region $\mathrm{W} 3(\mathrm{OH})$ by Sutton et al. (2004). No emission was detected in the $\mathrm{W} 3(\mathrm{OH}) /\left(\mathrm{H}_{2} \mathrm{O}\right)$ region at the sensitivity level achieved for their $104.1 \mathrm{GHz}$ observations.

The $216.9 \mathrm{GHz}$ spectrum shows an additional broader peak at approximately $-13 \mathrm{~km} \mathrm{~s}^{-1}$, coinciding with the velocity at which thermal methanol emission has previously been observed in this source (Val'tts et al. 1999; Salii \& Sobolev 2006). Although the signal to noise in each of these spectra is moderately low, subsequent SEST observations focusing on studying the thermal emission in this region have confirmed the presence of the maser emission in each of these two transitions (Salii \& Sobolev 2006). The observations of the 104.1 and 216.9 GHz transitions of Salii \& Sobolev (2006) were made in 2003 April, slightly less than three years after the observations reported here. In 2003, the $104.1 \mathrm{GHz}$ maser had a peak flux density of $\sim 3.8 \mathrm{Jy}$, consistent (within the relative uncertainties) with the flux density of the current SEST observations. In 2003, the $216.9 \mathrm{GHz}$ transition had a peak flux density of $\sim 10.3 \mathrm{Jy}$, significantly greater than the $4.2 \mathrm{Jy}$ reported in the current observations. Comparing the $216.9 \mathrm{GHz}$ spectrum in Figure 1 in Salii \& Sobolev (2006) with our Figure 1 shows that relative intensity of the maser and thermal components has not changed dramatically. Given that we do not expect to see any significant variations in the thermal emission, the observed difference in maser intensity is likely primarily due to calibration and/or pointing errors in the current observations rather than significant variations in the maser.

Salii \& Sobolev (2006) detected maser emission from the $231.3 \mathrm{GHz}\left(10_{2}-9_{3} \mathrm{~A}^{-}\right)$transition in $\mathrm{G} 345.01+1.79$ with a peak flux density of $\sim 2$ Jy. No maser emission was detected in our SEST observations; however, this is expected as they are significantly less sensitive (rms $1.5 \mathrm{Jy}$ ) than the observations of Salii \& Sobolev (2006).

We have fitted Gaussian profiles to both the maser emission and (where seen) the thermal emission for each transition. The results of these fits are summarized in Table 3. For all of the transitions it was necessary to fit more than one Gaussian profile to adequately describe the maser emission (i.e., residuals comparable with the noise). Many of the transitions have the strongest components at velocities close to -22.2 and $-21.4 \mathrm{~km} \mathrm{~s}^{-1}$ (e.g., 86.6, 86.9, and $104.1 \mathrm{GHz}$ ), while others show their strongest emission at a velocity of around $-21.7 \mathrm{~km} \mathrm{~s}^{-1}$ (e.g., 107.0, $108.9,156.6$, and $156.8 \mathrm{GHz}$ ). Even accounting for the relatively low signal to noise in some of the spectra, it is clear from this figure that although the maser emission in all transitions peaks at a velocity of around $-22 \mathrm{~km} \mathrm{~s}^{-1}$, it cannot be described by a single Gaussian component. Uncertainty in the absolute frequency calibration of the spectrometer and in the adopted rest frequencies of some of the transitions is approximately $0.2 \mathrm{~km} \mathrm{~s}^{-1}$, nevertheless, the difference in the shape and width of the emission at different frequencies requires the presence of two or more different maser components. 
Table 3

Gaussian Fitting of Methanol Maser Transitions in G 345.01+1.79

\begin{tabular}{|c|c|c|c|}
\hline $\begin{array}{l}\text { Transition } \\
(\mathrm{MHz})\end{array}$ & $\begin{array}{c}\text { Peak } \\
(\mathrm{Jy})\end{array}$ & $\begin{array}{l}\text { Velocity } \\
\left(\mathrm{km} \mathrm{s}^{-1}\right)\end{array}$ & $\begin{array}{l}\text { FWHM } \\
\left(\mathrm{km} \mathrm{s}^{-1}\right)\end{array}$ \\
\hline \multirow[t]{2}{*}{85.6} & $10.9(1.9)$ & $-22.10(0.01)$ & $0.51(0.07)$ \\
\hline & $4.9(2.0)$ & $-22.06(0.06)$ & $1.25(0.23)$ \\
\hline \multirow[t]{3}{*}{86.6} & $7.2(0.6)$ & $-22.25(0.05)$ & $0.66(0.09)$ \\
\hline & $6.0(0.5)$ & $-21.41(0.04)$ & $0.77(0.18)$ \\
\hline & $1.0(0.5)$ & $-20.48(0.22)$ & $0.52(0.43)$ \\
\hline \multirow[t]{2}{*}{86.9} & $6.8(0.5)$ & $-22.14(0.03)$ & $0.60(0.06)$ \\
\hline & $6.0(0.4)$ & $-21.32(0.03)$ & $0.82(0.10)$ \\
\hline \multirow[t]{3}{*}{104.1} & $2.8(1.1)$ & $-22.31(0.08)$ & $0.44(0.21)$ \\
\hline & $1.9(1.0)$ & $-21.35(0.16)$ & $0.64(0.41)$ \\
\hline & $1.1(0.5)$ & $-20.66(0.90)$ & $5.0(1.9)$ \\
\hline \multirow[t]{5}{*}{107.0} & $66(8)$ & $-21.85(0.12)$ & $0.98(0.14)$ \\
\hline & $29(8)$ & $-21.63(0.15)$ & $1.73(0.22)$ \\
\hline & $6(4)$ & $-22.14 .(0.42)$ & $3.11(0.52)$ \\
\hline & $2.0(0.4)$ & $-17.65(0.23)$ & $1.78(0.50)$ \\
\hline & $3.7(0.3)$ & $-12.65(0.11)$ & $2.55(0.27)$ \\
\hline \multirow[t]{4}{*}{108.9} & $2.3(0.8)$ & $-22.36(0.16)$ & $0.47(0.17)$ \\
\hline & $8.3(0.4)$ & $-21.67(0.14)$ & $0.82(0.08)$ \\
\hline & $4.0(1.3)$ & $-14.65(0.94)$ & $3.80(1.16)$ \\
\hline & $7.3(2.9)$ & $-12.62(0.14)$ & $2.43(0.33)$ \\
\hline \multirow[t]{3}{*}{156.6} & $10.5(3.6)$ & $-22.49(0.06)$ & $0.48(0.15)$ \\
\hline & $27.6(1.2)$ & $-21.79(0.04)$ & $0.95(0.11)$ \\
\hline & $10.5(0.6)$ & $-12.80(0.22)$ & $3.31(0.24)$ \\
\hline \multirow[t]{2}{*}{156.8} & $45(1.8)$ & $-21.68(0.03)$ & $1.36(0.06)$ \\
\hline & $6.6(0.6)$ & $-12.80(0.22)$ & $1.99(0.51)$ \\
\hline \multirow[t]{3}{*}{216.9} & $3.6(0.3)$ & $-12.82(0.10)$ & $2.31(0.23)$ \\
\hline & $4.2(0.8)$ & $-22.50(0.10)$ & $0.49(0.11)$ \\
\hline & $2.0(0.5)$ & $-22.02(0.23)$ & $2.29(0.43)$ \\
\hline
\end{tabular}

Note. Formal uncertainty from fitting in parenthesis.

\section{DISCUSSION}

G $345.01+1.79$ has been observed at 14 of the 15 class II methanol maser transition (or transition groups) frequencies listed in Table 1 . The exception is the $8_{2}-9_{1} \mathrm{~A}^{-}(29.0 \mathrm{GHz})$ transition for which there is only one published study (Wilson et al. 1993). Including the two new class II transitions reported

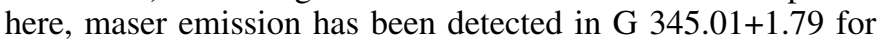
15 of the 16 known class II transitions (or transition groups) observed in this source, the exception being the $9_{2}-10_{1} \mathrm{~A}^{+}$ $23.1 \mathrm{GHz}$, for which the sensitive observations of Cragg et al. (2004) placed a $3 \sigma$ upper limit of $0.5 \mathrm{Jy}$. Some other well-studied $\mathrm{H}$ II regions (such as $\mathrm{W} 3(\mathrm{OH})$ and NGC 6334F) also show a large number of class II methanol masers, but no others exhibit as many as G $345.01+1.79$. It is not clear why $\mathrm{G} 345.01+1.79$ is such an exceptional source. Norris et al. (1993) identified two distinct class II methanol maser sites (separated by 19", with non-overlapping velocity ranges) in this general region. G 345.010+1.792 has methanol emission covering the velocity range -24 to $-16 \mathrm{~km} \mathrm{~s}^{-1}$, while $\mathrm{G} 345.012+1.797$ masers cover -16 to $-10 \mathrm{~km} \mathrm{~s}^{-1}$ (Caswell 2009). G 345.010+1.792 is associated with a UCH II region, and all of the rare/weak methanol maser transitions are associated with this source. Breen et al. (2010) detected three water masers in this region, one associated with each maser site and a third which is not associated with any other known maser transitions. Interestingly, the weakest water maser in the region is associated with the strongest methanol maser site ( G 345.010+1.792), which also has an associated OH maser (Caswell 1998), while the secondary methanol maser site is associated with the strongest water maser. In this Letter, we are focusing purely on the strongest methanol maser site (G 345.010+1.792).

In general, the strongest emission from class II methanol maser transitions in this source is at velocities near $-22 \mathrm{~km} \mathrm{~s}^{-1}$. In contrast, when it was first observed in the early 1990s, the peak velocity of the $6.7 \mathrm{GHz}$ methanol maser was at $-17 \mathrm{~km} \mathrm{~s}^{-1}$ (Caswell et al. 1995b). The intensity of that spectral feature has declined over the intervening 20 years (see, e.g., Ellingsen et al. 2004; Caswell et al. 2010), and the peak of the $6.7 \mathrm{GHz}$ maser emission is now at $\sim-21 \mathrm{~km} \mathrm{~s}^{-1}$. The $19.9 \mathrm{GHz}$ methanol maser is now the only transition which peaks at $-17 \mathrm{~km} \mathrm{~s}^{-1}$ (Ellingsen et al. 2004), and in contrast to the other rare/weak methanol maser transitions no emission is observed at velocities less than $-19 \mathrm{~km} \mathrm{~s}^{-1}$.

The complex, nonlinear relationship between the physical conditions of the masing gas and the intensity of the observed maser means that it is generally not possible to uniquely infer the former from the latter. Where more than one maser transition is observed from the same volume of gas, it must be the case that a complete theoretical maser model will be able to find conditions that simultaneously invert all of the detected transitions in the observed intensity ratios. Cragg et al. (2001) used information on nine class II methanol maser transitions, collected with a variety of instruments, over a period of several years, to constrain the physical conditions in G 345.01+1.79, using the model of Sobolev et al. (1997a). They found the observed ratio of maser intensities to be consistent with cool gas (30K) at a density of $10^{6} \mathrm{~cm}^{-3}$ with a methanol column density of $5 \times 10^{17} \mathrm{~cm}^{-2}$, being pumping by the radiation from warm $(175 \mathrm{~K})$ dust.

Modeling such as that undertaken by Cragg et al. (2001) requires a number of assumptions. In particular, it is assumed that the emission from each transition arises from the same region of gas and that the emitting region is of the same size for each transition. Where information is collated from observations made with different instruments over a period of years, it is also necessary to ignore the effects of temporal variability. For G345.01+1.79, Minier (2004) showed that the 6.7, 12.2, 85.5, and $86.6 \mathrm{GHz}$ transitions are all coincident to within $1^{\prime \prime}$ (the relative positional accuracy of the observations), and where it has been possible to test, the assumption that the different maser transitions are cospatial with greater precision this appears to be generally true (Menten et al. 1992; Norris et al. 1993; Sutton et al. 2001). The assumption that the emitting regions are of the same size in each transition is not likely to be correct, but it requires very long baseline interferometry observations to make the necessary measurements, and at present that is only practical for the 6.7 and $12.2 \mathrm{GHz}$ transitions. Although the variability of class II methanol masers is not as extreme as that observed for water masers, variations on timescales of months or years are common (Caswell et al. 1995a; Goedhart et al. 2004). For the weaker millimeter methanol masers, variability is also expected, possibly even to a greater extent due to the lower degree of saturation in these transitions, and evidence of variations in the millimeter masers in $\mathrm{G} 345.01+1.79$ have been observed (Ellingsen et al. 2003).

Of the many thousands of methanol transitions which fall at centimeter or millimeter wavelengths, a small subset (around 20) exhibits pronounced maser characteristics. Many of the maser lines can be grouped into series with the same patterns of $J$ and $K$ quantum numbers. For example, the $5_{1}-6_{0} \mathrm{~A}^{+}$maser at $6.7 \mathrm{GHz}$ is part of a series of $J_{1}-(J+1)_{0} \mathrm{~A}^{+}$masers including the $3_{1}-4_{0} \mathrm{~A}^{+}$maser at $107.0 \mathrm{GHz}$ and the $2_{1}-3_{0} \mathrm{~A}^{+}$ maser at $156.6 \mathrm{GHz}$. This is consistent with the models that 
also predict maser action in the $4_{1}-5_{0} \mathrm{~A}^{+}$transition at $57.0 \mathrm{GHz}$ (which cannot be observed by ground-based telescopes due to atmospheric absorption by molecular oxygen at this frequency). One can think of the $K=1$ ladder of energy levels as being slightly overpopulated with respect to the nearby $K=0$ energy levels, with the resulting population inversions being responsible for the maser action. The new maser transitions detected in the current work are the first members of two new series of methanol transitions to exhibit maser action and as such represent a more stringent test of the models. Our detection of maser action in the $5_{1}-4_{2} \mathrm{E}$ transition at $216.9 \mathrm{GHz}$ is consistent with the modeling of Sobolev et al. (1997b) and Cragg et al. (2005), which also include the $3_{1}-2_{2} \mathrm{E} 120.2 \mathrm{GHz}, 4_{1}-3_{2} \mathrm{E}$ 168.6, and $6_{1}-5_{2}$ E $265.3 \mathrm{GHz}$ transitions from the same series in the list of maser candidates. The newly observed $13_{-3}-12_{-4} \mathrm{E}$ maser at $104.1 \mathrm{GHz}$ is part of the same series as the $11_{-3}-10_{-4} \mathrm{E}$ $7.3 \mathrm{GHz}$ and $12_{-3}-11_{-4}$ E $55.7 \mathrm{GHz}$ transitions, both of which were identified as class II methanol maser candidates in these models. In the models presented in these papers, the $104.1 \mathrm{GHz}$ maser candidate was below the threshold intensity for listing, indicating that its excitation to observable levels requires more extreme conditions.

Detailed modeling of the methanol masers in G345.01+1.79 is beyond the scope of this Letter and will be the subject of a future publication. In addition to the data presented here, previous and subsequent observations with SEST and other instruments have obtained detections and limits on the emission from more than three dozen known or potential class II methanol transitions. Here we restrict ourselves to a preliminary exploration which utilizes the data from these observations, in combination with published 6.7 and $12.2 \mathrm{GHz}$ spectra, following the approach outlined in Cragg et al. (2001). The significant differences in the detailed spectral structure of the methanol maser emission in different transitions near velocities of $-22 \mathrm{~km} \mathrm{~s}^{-1}$ demonstrates the presence of multiple, partially blended maser components. This implies that within G 345.01+1.79 there are multiple sites with differing physical conditions which are giving rise to emission in rare methanol transitions. Hence, it is unlikely that any single model fit will be able to explain the observed intensities of all of the transitions, and this is indeed the case.

To adequately explain the observed (and the one nondetected) class II methanol transitions in G 345.01+1.79 using the Sobolev et al. (1997a) model, we require emission from at least two regions with similar/overlapping line-of-sight velocities but different physical parameters. This is inferred from the differences in the line profiles of the observed transitions (e.g., Figure 2 shows that the 86.6, 86.9, and $104.1 \mathrm{GHz}$ transitions are double-peaked, while other transitions are single-peaked) and in their peak velocities (see Table 3). Applying models which assume that emission in all the transitions is cospatial cannot produce a single, self-consistent solution in cases such as this. For example, in G 345.01+1.79 we observe both maser transitions that require low densities (e.g., the 86.6 and $86.9 \mathrm{GHz}$ ) and transitions that prefer higher densities (e.g., the 85.6 and 104.1 GHz) for their efficient excitation. We suggest that the reason for $\mathrm{G} 345.01+1.79$ exhibiting maser emission in an unusually large number of different methanol transitions may be that the coexistence of masing regions covering such a broad range of physical conditions is rare.

\section{CONCLUSIONS}

We have discovered two new class II methanol maser transitions toward the G 345.01+1.79 star formation region. The emis- sion from these transitions is relatively weak in G 345.01+1.79 and it is likely that only a small number of high-mass star formation regions will show maser emission in these transitions. G 345.01+1.79 clearly has great potential for helping us to study the physical conditions in high-mass star formation regions at high resolution, through observations of the large number of class II methanol maser transitions. The primary limitations on the current observations are due to relatively low signal to noise and spatial blending. High-resolution observations of the weaker millimeter methanol masers to obtain detailed information on their distribution, to constrain the spot size, and to determine whether the different transitions are coincident have recently become much more feasible with the commencement of Atacama Large Millimeter Array operations. Such observations offer the prospect of unlocking the potential of multitransition maser observations and providing important insights into the processes which occur in high-mass star formation regions.

This research has made use of NASA's Astrophysics Data System Abstract Service. Travel to SEST for observations was provided under the access to major research facilities program which is supported by the Commonwealth of Australia under the International Science Linkages program. A.M.S. was partly supported by the Russian state contract No. 14.518.11.7064 and the Russian Foundation for Basic Research (grants 10-0200589-a and 11-02-01332-a). D.M.C. and P.D.G. acknowledge financial support for this work from the Australian Research Council.

\section{REFERENCES}

Batrla, W., Matthews, H. E., Menten, K. M., \& Walmsley, C. M. 1987, Nature, 326, 49

Breen, S. L., Caswell, J. L., Ellingsen, S. P., \& Phillips, C. J. 2010, MNRAS, 406, 1487

Caswell, J. L. 1998, MNRAS, 297, 215

Caswell, J. L. 2009, PASA, 26, 454

Caswell, J. L., Fuller, G. A., Green, J. A., et al. 2010, MNRAS, 404, 1029

Caswell, J. L., Fuller, G. A., Green, J. A., et al. 2011, MNRAS, 417, 1964

Caswell, J. L., Vaile, R. A., \& Ellingsen, S. P. 1995, PASA, 12, 37

Caswell, J. L., Vaile, R. A., Ellingsen, S. P., Whiteoak, J. B., \& Norris, R. P. 1995, MNRAS, 272, 96

Caswell, J. L., Yi, J., Booth, R. S., \& Cragg, D. M. 2000, MNRAS, 313, 599

Cragg, D. M., Sobolev, A. M., Caswell, J. L., Ellingsen, S. P., \& Godfrey, P. D. 2004, MNRAS, 351, 1327

Cragg, D. M., Sobolev, A. M., Ellingsen, S. P., et al. 2001, MNRAS, 323, 939

Cragg, D. M., Sobolev, A. M., \& Godfrey, P. D. 2002, MNRAS, 331, 521

Cragg, D. M., Sobolev, A. M., \& Godfrey, P. D. 2005, MNRAS, 360, 533

Cyganowski, C. J., Brogan, C. L., Hunter, T. R., \& Churchwell, E. 2009, ApJ, 702,1615

Ellingsen, S. P. 2006, ApJ, 638, 241

Ellingsen, S. P., Breen, S. L., Sobolev, A. M., et al. 2011, ApJ, 742, 109

Ellingsen, S. P., Cragg, D. M., Lovell, J. E. J., et al. 2004, MNRAS, 354, 401

Ellingsen, S. P., Cragg, D. M., Minier, V., Muller, E., \& Godfrey, P. D. 2003, MNRAS, 344, 73

Ellingsen, S. P., von Bibra, M. L., McCulloch, P. M., et al. 1996, MNRAS, 280, 378

Green, J. A., Caswell, J. L., Fuller, G. A., et al. 2010, MNRAS, 409, 913

Goedhart, S., Gaylard, M. J., \& van der Walt, D. J. 2004, MNRAS, 355, 553

Haschick, A. D., Baan, W. A., \& Menten, K. M. 1989, ApJ, 339, 949

Kurtz, S., Hofner, P., \& Álvarez, C. V. 2004, ApJS, 155, 149

Menten, K. M. 1991a, in ASP Conf. Ser. 16, Atoms, Ions and Molecules: New Results in Spectral Line Astrophysics, ed. A. D. Haschick \& P. T. P. Ho (San Francisco, CA: ASP), 119

Menten, K. M. 1991b, ApJ, 380, L75

Menten, K. M., Reid, M. J., Pratap, P., Moran, J. M., \& Wilson, T. L. 1992, ApJ, 401, L39 
Minier, V. 2004, in IAU Symp. 221, Star Formation at High Angular Resolution, ed. M. G. Burton, R. Jayawardhana, \& T. L. Bourke (Cambridge: Cambridge Univ. Press), 275

Minier, V., Ellingsen, S. P., Norris, R. P., \& Booth, R. S. 2003, A\&A, 403, 1095

Norris, R. P., Caswell, J. L., Wellington, K. J., McCutcheon, W. H., \& Reynolds, J. E. 1988, Nature, 335, 149

Norris, R. P., Whiteoak, J. L., Caswell, J. L., Wieringa, M. H., \& Gough, R. G. 1993, ApJ, 412, 222

Phillips, C. J., Norris, R. P., Ellingsen, S. P., \& McCulloch, P. M. 1998, MNRAS, 300, 1131

Salii, S. V., \& Sobolev, A. M. 2006, Astron. Rep., 50, 965

Salii, S. V., Sobolev, A. M., Kalinina, N. D., et al. 2003, in Communications of the Konkoly Observatory, The Interaction of Stars with Their Environment II, ed. Cs. Kiss, M. Kun, \& V. Könyves (Budapest, Hungary: Hungarian Academy of Science), 75

Sastry, K. V. L. N., Lees, R. M., \& De Lucia, F. C. 1984, J. Mol. Spectrosc., 103,486

Slysh, V. I., Kalenskii, S. V., \& Val'tts, I. E. 1995, ApJ, 442, 668

Sobolev, A. M., Cragg, D. M., \& Godfrey, P. D. 1997a, A\&A, 324, 211

Sobolev, A. M., Cragg, D. M., \& Godfrey, P. D. 1997b, MNRAS, 288, L39

Sutton, E. C., Sobolev, A. M., Ellingsen, S. P., et al. 2001, ApJ, 554, 173
Sutton, E. C., Sobolev, A. M., Salii, S. V., et al. 2004, ApJ, 609, 231

Tsunekawa, S., Ukai, T., Toyama, A., \& Takagi, K. 1995, Microwave Frequencies of the $\mathrm{CH}_{3} \mathrm{OH}$ Molecule in the Frequency Range from 7 to $200 \mathrm{GHz}$, Report for the Grant-in-aid for Scientific Research on Priority Areas (Interstellar Matter, 1991-1994) of the Ministry of Education, Science, and Culture, Japan. Toyama University, Japan

Val'tts, I. E., Dzura, A. M., Kalenskii, S. V., et al. 1995, A\&A, 294, 825

Val'tts, I. E., Ellingsen, S. P., Slysh, V. I., et al. 1999, MNRAS, 310, 1077

Voronkov, M., Sobolev, A., Ellingsen, S., Ostrovskii, A., \& Alakoz, A. 2005, Ap\&SS, 295, 217

Walsh, A. J., Burton, M. G., Hyland, A. R., \& Robinson, G. 1998, MNRAS, 301,640

Walsh, A. J., Macdonald, G. H., Alvey, N. D. S., Burton, M. G., \& Lee, J.-K. 2003, A\&A, 410, 597

Wilson, T. L., Huettemeister, S., Dahmen, G., \& Henkel, C. 1993, A\&A, 268 249

Wilson, T. L., Walmsley, C. M., Menten, K. M., \& Hermsen, W. 1985, A\&A, 147, L19

Wilson, T. L., Walmsley, C. M., Snyder, L. E., \& Jewell, P. R. 1984, A\&A, 134, L7

Xu, Y., Li, J. J., Hachisuka, K., et al. 2008, A\&A, 485, 729 